\title{
THE PRINCIPAL MATRICES OF A RIEMANN MATRIX*
}

BY A. A. ALBERT

1. Introduction. A matrix $\omega$ with $p$ rows and $2 p$ columns of complex elements is called a Riemann matrix if there exists a rational $2 p$-rowed skew-symmetric matrix $C$ such that

$$
\omega C \omega^{\prime}=0, \quad \pi=i \omega C \bar{\omega}^{\prime}
$$

is positive definite. The matrix $C$ is called a principal matrix of $\omega$ and it is important in algebraic geometry to know what are all principal matrices of $\omega$ in terms of a given one. In the present note I shall solve this problem.

2. Principal Matrices. A rational $2 p$-rowed square matrix $A$ is called a projectivity of $\omega$ if

$$
\alpha \omega=\omega A
$$

for a $p$-rowed complex matrix $\alpha$. The Riemann matrices $\omega$ have recently $\dagger$ been completely classified in terms of their projectivities; so we may regard all the projectivities $A$ of $\omega$ as known.

A projectivity $A$ is called symmetric if $C A^{\prime} C^{-1}=A$. Let $A$ be a symmetric projectivity so that if $B=A C$, then $B^{\prime}=(A C)^{\prime}$ $=-C A^{\prime}=-A C=-B$ is a skew-symmetric matrix. Then $i A C$ is Hermitian and so must be

$$
\delta=\omega(i A C) \bar{\omega}^{\prime}=\alpha\left(i \omega C \bar{\omega}^{\prime}\right)=\alpha \pi .
$$

Now $\pi$ is positive definite so that $\pi=\rho \bar{\rho}^{\prime}$, where $\rho$ is nonsingular. Then $\pi^{-1}=\left(\bar{\rho}^{\prime}\right)^{-1} \rho^{-1}=\bar{\sigma}^{\prime} \sigma$ with $\sigma$ non-singular. Hence $\alpha=\delta \pi^{-1}=\delta \bar{\sigma}^{\prime} \sigma$ and

$$
\sigma \alpha \sigma^{-1}=\sigma \delta \bar{\sigma}^{\prime}
$$

The matrix $\sigma \delta \bar{\sigma}^{\prime}$ is evidently Hermitian and it is well known that then $\sigma \delta \bar{\sigma}^{\prime}$ and the similar matrix $\alpha$ have only simple ele-

* Presented to the Society, September 7, 1934.

$\dagger$ See my paper $A$ solution of the principal problem in the theory of Riemann matrices, Annals of Mathematics, October, 1934. 
mentary divisors and all real characteristic roots. Thus $\alpha=\beta \gamma \beta^{-1}$, where $\gamma$ is a real diagonal matrix.

Write

$$
\Omega=\left(\frac{\omega}{\omega}\right),
$$

so that, as is well known, and may easily be computed,

$$
A=\Omega^{-1}\left(\begin{array}{ll}
\alpha & 0 \\
0 & \bar{\alpha}
\end{array}\right) \Omega=\Lambda \Gamma \Lambda^{-1}
$$

where

$$
\Gamma=\left(\begin{array}{ll}
\gamma & 0 \\
0 & \gamma
\end{array}\right), \quad \Lambda=\Omega^{-1}\left(\begin{array}{ll}
\beta & 0 \\
0 & \bar{\beta}
\end{array}\right)
$$

Then $A$ is similar to the real diagonal matrix $\Gamma$ and we have proved the following theorem.*

THEOREM 1. A symmetric projectivity of a Riemann matrix has all simple elementary divisors and all real characteristic roots.

We may now determine all principal matrices of a given Riemann matrix $\omega$ with a given principal matrix $C$. Let $B$ be a second principal matrix of $\omega$ so that $\omega B \omega^{\prime}=0$. It is well known that $B C=A$ is a projectivity of $\omega$. In fact $\alpha \omega=\omega A$, where $\alpha=\delta \pi^{-1}$ is defined by (3). Moreover $B^{\prime}=-B$, so that

$$
(A C)^{\prime}=C^{\prime} A^{\prime}=-C A^{\prime}=-A C,
$$

and $C A^{\prime} C^{-1}=A$. Hence $A=B C^{-1}$ is a symmetric projectivity of $\omega$.

The matrix $\delta=i \omega B^{\prime} \bar{\omega}$ is positive definite if $B$ is a principal matrix of $\omega$. Hence $\sigma \delta^{\prime} \sigma^{\prime}$ is positive definite and has all positive characteristic roots. The matrices $\alpha$ and $\gamma$ defined above are similar to $\sigma \alpha \sigma^{-1}=\sigma \delta \bar{\sigma}^{\prime}$ and have the same characteristic roots, so that the diagonal matrix $\Gamma$, whose diagonal elements are these characteristic roots repeated, has all positive diagonal elements. Then $A$, which is similar to $\Gamma$, has all positive characteristic roots.

Conversely, let $A$ be a symmetric projectivity of $\omega$ with all positive characteristic roots. Then $\Gamma$ has all positive diagonal

* The proof by the use of (4) was suggested by certain analogous considerations of N. Jacobson. 
elements, $\alpha$ has all positive characteristic roots and so has $\sigma \alpha \sigma^{-1}=\sigma \delta \bar{\sigma}^{\prime}$. But $\sigma \delta \bar{\sigma}^{\prime}$ is an Hermitian matrix with characteristic roots all positive. Then $\sigma \delta \bar{\sigma}^{\prime}$ is positive definite and so is $\delta=i \omega A C \omega^{\prime}$. Moreover, if $B=A C$, then

$$
\omega B \omega^{\prime}=\omega A C \omega^{\prime}=\alpha \omega C \omega^{\prime}=0
$$

and $B$ is a principal matrix of $\omega$. We have proved the following result.

THEOREM 2. Let $\omega$ be a Riemann matrix with principal matrix $C$ and let $A$ range over the set of all symmetric projectivities of $\omega$ which have positive characteristic roots. Then a rational matrix $B$ is a principal matrix of $\omega$ if and only if $B=A C$ with $A$ in the above set.

3. Pure Riemann Matrices of the First Kind. The problem of determining what projectivities of $\omega$ are symmetric with all characteristic roots positive is, in general, a complicated one. We may nevertheless solve this problem for the case where $\omega$ is a pure Riemann matrix of the first kind.

The multiplication algebra of a pure Riemann matrix is a division algebra $D$. The centrum of $D$ is a field represented by a field $R(S)$ of all polynomials with rational coefficients of a projectivity $S$ of $\omega$. Algebra $D$ is of the first or second kind according as $S$ is or is not symmetric.

If $D$ is of the first kind, then I have proved* that every projectivity of $\omega$ has the form $p(S)$ in $R(S)$ or the form

$$
\alpha_{1}+\alpha_{2} X+\alpha_{3} Y+\alpha_{4} X Y,
$$

with $\alpha_{1}, \cdots, \alpha_{4}$ in $R(S)$, such that

$$
Y X=-X Y, \quad X^{2}=\xi, \quad Y^{2}=\eta, \quad(\xi, \eta \text { in } R(S)) .
$$

The order of the set of all symmetric projectivities of $\omega$ is its singularity index $k$. If $S$ is symmetric and $R(S)$ has order $t$, then $k=t$ or $k=3 t$ according as we may not or may take both $X$ and $Y$ symmetric, while $k=t$ if $D$ is equivalent to $R(S)$.

Let first $k=t$ so that every symmetric projectivity of $\omega$ is in $R(S)$, and let the characteristic roots of $S$ be $\sigma_{1}, \cdots, \sigma_{t}$. Then

\footnotetext{
* Annals of Mathematics, vol. 33 (1932), pp. 311-318.
} 
if $A=p(S)$, the characteristic roots of $A$ are $p\left(\sigma_{j}\right)$ and we have the following theorem.

TheOREM 3. Let $\omega$ be a pure Riemann matrix of the first kind with projectivity algebra $D_{0}$ over $R(S)$ having singularity index $k=t$. Then the principal matrices of $\omega$ are the matrices

$$
p(S) C,
$$

where $p(S)$ is a polynomial in $S$ with rational coefficients such that

$$
p\left(\sigma_{j}\right)>0, \quad(j=1, \cdots, t) .
$$

Next let $k=3 t$ so that every symmetric projectivity of $\omega$ has the form

$$
A=p_{1}(S)+p_{2}(S) X+p_{3}(S) Y .
$$

Then $A$ satisfies the equation in an indeterminate $\alpha$

$$
\left[\alpha-p_{1}(S)\right]^{2}=\left[p_{2}(S)\right]^{2} \xi+\left[p_{3}(S)\right]^{2} \eta .
$$

Hence the characteristic roots of $A$ are the numbers

$$
p_{1}\left(\sigma_{j}\right) \pm\left\{\left[p_{2}\left(\sigma_{j}\right)\right]^{2} \xi\left(\sigma_{j}\right)+\left[p_{3}\left(\sigma_{j}\right)\right]^{2} \eta\left(\sigma_{j}\right)\right\}^{1 / 2} .
$$

Since $X$ and $Y$ are symmetric we have the well known trivial result

$$
\xi\left(\sigma_{j}\right)>0, \quad \eta\left(\sigma_{j}\right)>0 .
$$

But then the characteristic roots of $A$ are all positive if and only if

$$
p_{1}\left(\sigma_{j}\right)>\left\{\left[p_{2}\left(\sigma_{j}\right)\right]^{2} \xi\left(\sigma_{j}\right)+\left[p_{3}\left(\sigma_{j}\right)\right]^{2} \eta\left(\sigma_{j}\right)\right\}^{1 / 2} .
$$

We have proved the following theorem.

THEOREM 4. Let $\omega$ be pure with singularity index $k=3$ tand let $p_{1}(S), p_{2}(S), p_{3}(S)$ be polynomials in $S$ with rational coefficients. Then every principal matrix of $\omega$ is given by the set of matrices

$$
\left[p_{1}(S)+p_{2}(S) X+p_{3}(S) Y\right] C,
$$

with $p_{1}, p_{2}, p_{3}$ chosen so that (14) holds.

The University of Chicago 